\title{
14
}

\section{Bounds on the Effects of Correlation in a Stable MMPP/MMPP/1/N Queue: An Asymptotic Approach*}

\author{
Demetres Kouvatsos ${ }^{\mathrm{a}}$, Rod Fretwell ${ }^{\mathrm{a}}$ and János Sztrik ${ }^{\mathrm{b}}$ \\ ${ }^{a}$ Computer Systems Modelling Research Group, \\ University of Bradford, \\ Bradford BD7 1DP, UK \\ ${ }^{\mathrm{b}}$ Department of Mathematics, \\ University of Debrecen, Hungary
}

Correlation and burstiness are important in characterisating ATM traffic and modelling ATM switch architectures. The solution space of a system of correlated processes comprises regions of low intensity and of high intensity, in which correlation has little effect, and a region of intermediate intensity, in which correlation is significant. However, the locations of such regions have not previously been defined.

In this paper an asymptotic queueing theoretic method is applied to the performance analysis of a finite capacity, single server queue evolving within a randomly changing environment. All stochastic times in the system are considered to be distributed exponentially while the arrival and service rates are subject to random fluctuations. It is shown that, for a stable MMPP/MMPP $/ 1 / \mathrm{N}$ queue, the regions are clearly defined and the location of the boundaries are given as simple, closed form expressions. The method also reveals that MMPP/MMPP/1/N systems comprise families which are related by common features of the MMPP model parameters. The behaviour of all members of one family is practically identical in the regions of low intensity and of high intensity but may be markedly divergent in the region of intermediate intensity.

Comments on further extension to the work and its applicability to performance analysis of multi-buffered ATM switch architectures are given.

\section{Introduction}

ATM traffic is commonly described as being bursty and correlated.

A variety of traffic models (such as Markov modulated processes) have been proposed which are deemed to capture the burstiness and correlation of streams of ATM traffic and of superposition of multiple streams. These traffic models have been used to study performance of ATM multiplexers, switches and networks - predominantly in simulation studies or by numerical solutions to queueing system models. Both simulation and numerical methods are practically limited by the effects of "state space explosion": in simulation, as the number of traffic streams increase, run times become prohibitively long and, even for small models, it is difficult to achieve the precision required for quality of

\footnotetext{
${ }^{*}$ This work is supported by the Engineering and Physical Sciences Research Council (EPSRC), UK, under grant GR/H/18609 and by the Hungarian National Science Foundation, Hungary, under grant OTKA-1648
} 
service parameters (e.g. cell loss probability $\sim 10^{-12}$ ); methods for numerical solution of queueing system models become unstable with increase in state space and are satisfactory only for relatively small models (e.g. $16 \times 16$ shared buffer switch). Further, whilst the choice of traffic model parameters is arbitrary to a degree, sensitivity of results to variations in parameters cannot be determined readily from either simulation or numerical methods.

Queueing systems with ATM traffic models as arrival processes do not lend themselves to tractable analysis, except in the most simple cases. Compared with the closed form expressions which give insight into behaviour of systems comprising processes free of correlation, useful analytic results are lacking for systems having correlated processes. Consequently, there is fundamental need for simple, cost effective approximations.

Intuitively, it appears that correlation should have little effect at extremes of loading. At high intensity, when the buffer is almost always full and almost all arrivals are blocked, there is little scope for correlation between arrivals to affect system behaviour. At low intensity, when almost all arrivals "see" an empty system, there can be little interaction between arrivals.

Consideration of that intuitive argument raises the issues which are addressed in this paper.

- Ranges of model parameters for which system behaviour is not significantly affected by correlation.

- Relationships between model parameters in the ranges for which correlation is not significant. (Observe that, if correlation be significant over only limited ranges of intensity, there must be families of systems which have convergent behaviour in those ranges.)

- Consideration of whether bounds on system behaviour in the regions where correlation has significant effect may be derived by extrapolation from the behaviour in the regions in which system behaviour is not significantly affected by correlation.

There are no previously known methods (neither theoretical nor heuristic) which identify either regions in which correlation be insignificant or families of related processes.

In this paper, asymptotic techniques (to reduce effects of correlation) and stochastic arguments (which are valid when correlation is not significant) are employed to produce simple performance approximations. These approximations are then used to define the regions in which correlation is not significant and the corresponding families of systems having the same behaviour in those regions. The particular queueing system considered is a finite buffer queue with censored Markov Modulated Poisson Process (MMPP) arrivals and MMPP service: the MMPP has been used as a model for ATM traffic in continuous time; in this paper, choice of the MMPP enables us to focus upon the proposed technique, without additional complications involved in other models or in discrete time systems.

Anisimov and Sztrik [1] presented a theorem, due to Anisimov et al. [2], and applications of that theorem to the asymptotic analysis of finite source queueing systems evolving in random environments, i.e. in which arrival rates and service rates are modulated by Markov chains. The analysis is of the asymptotic behaviour of the queueing system under the assumption of fast service, i.e. as the service rates increase without limit. Subsequently, Sztrik [3,4] presented applications of the theorem under the assumption of fast arrivals, in which the analysis is of the asymptotic behaviour of the queueing system 
as the arrival rates increase without limit. All the applications presented by Anisimov and by Sztrik are variants of the Machine Repairman model in a random environment and the results have been validated in a stable environment only - not for Markov modulated processes.

In this paper, asymptotic approximations are derived, both for fast service and for fast arrivals, applicable to MMPP/MMPP/1/N queueing systems. The ranges of validity of the approximations are established and the results validated against exact calculations for sample MMPP/MMPP/1/N queueing systems.

Thus, in addition to the principal contributions of this paper, it also shows

- new asymptotic results,

- first application relevant to ATM,

- first application utilizing both fast service and fast arrivals,

- first validation in random environments.

In section 2, an MMPP/MMPP/1/N queueing system is described as an $M / M / 1 / N$ system evolving in a randomly changing environment. An embedded Markov chain is defined and the state transition probabilities are derived.

In section 3 , the asymptotic forms of the state transition probabilities are derived under the assumption of fast service. Anisimov's Theorem is presented and applied to yield the limiting distribution of the system sojourn time in a defined subset of the state space.

The method is then applied under the assumption of fast arrivals in section 4 to yield the limiting distribution of the busy period.

The results of sections 3 and 4 are combined in section 5 under a simplifying assumption to yield particular asymptotic approximations to utilisation which readily lead to simple closed form expressions for the positions of the boundaries between regions in which correlation has significant effect and those in which it does not. Derivation of accurate asymptotic approximations to utilisation, queue length distribution, etc. - which is not the principal purpose of this paper - is deferred to an appendix.

Results of comparisons between the asymptotic approximations and exact calculations for utilisation in simple MMPP/MMPP/1/N systems are presented in section 6 .

Finally, conclusions follow in section 7 .

\section{Queueing Model - MMPP/MMPP/1/N with Censored Arrivals Process}

Consider a simple finite buffer queueing system with a single server in which both the arrivals process and the service process are Markov Modulated Poisson Processes and the arrivals process is censored: arrivals to a full buffer are simply lost.

A Markov Modulated Poisson Process (MMPP) may be described as being a Poisson process which is evolving in a randomly changing environment. The phase of the MMPP is the state of the governing environmental Markov chain and, at any instant, the parameter of the Poisson process is determined by the phase of the governing environmental Markov chain. Thus, a MMPP/MMPP/1/N queueing system may be viewed as a $M / M / 1 / N$ system in which the arrivals process is modulated by one environmental Markov chain and the service process is modulated by another environmental Markov chain. 
Further, as is well known, the $\mathrm{M} / \mathrm{M} / 1 / \mathrm{N}$ system with either restricted or censored arrival process is equivalent to a closed 2-stage cyclic queueing network in which the arrivals to the $\mathrm{M} / \mathrm{M} / 1$ queue (server $\Sigma_{2}$ ) are just the departures from the preceding server $\left(\Sigma_{1}\right)$ and the population is of fixed size N. In general, the closed 2-stage cyclic queueing network is the dual of a finite buffer queueing system with restricted arrivals process. The equivalence of censored and restricted arrivals results from the memoryless property of the Poisson arrivals. Similar considerations apply in the case considered here. When the arrivals be blocked by a full buffer (at server $\Sigma_{2}$ in figure 1), the environment $\left(\xi_{A}\right.$ in figure 1) which governs the arrivals process is not frozen. Thus, because (in each phase) the arrivals process is Poisson (therefore memoryless), at the instant at which the block on arrivals is removed, the restricted arrivals process is in exactly the same condition as a censored arrivals process would be. Then, for MMPP arrivals, restricted arrivals and censored arrivals are the same.

Thus, to analyse the MMPP/MMPP/1/N queueing system, we may consider the (dual) system shown in figure 1 in which

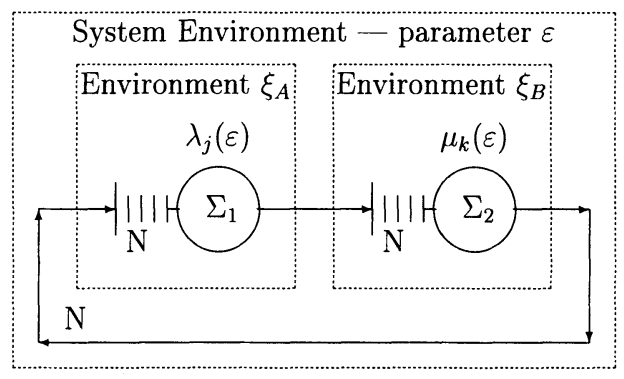

Figure 1. Closed 2-stage Cyclic Queueing Network, with fixed population N, and Markov Modulated Poisson Processes at each Station.

arrival rates are governed by an ergodic Markov chain $\left(\xi_{A}(t), t \geq 0\right)$ with state space $\left\{1, \ldots, r_{A}\right\}$, transition rate matrix $\left\{a_{j l}, j, l=1, \ldots, r_{A}\right\}$ and steady state distribution $\left\{\pi_{A}(j), j=1, \ldots, r_{A}\right\}$ and, when $\xi_{A}$ is in state $j$, the arrival rate is $\lambda_{j}(\varepsilon)$,

departure rates are governed by an ergodic Markov chain $\left(\xi_{B}(t), t \geq 0\right)$ with state space $\left\{1, \ldots, r_{B}\right\}$, transition rate matrix $\left\{b_{k q}, k, q=1, \ldots, r_{B}\right\}$ and steady state distribution $\left\{\pi_{B}(k), k=1, \ldots, r_{B}\right\}$ and, when $\xi_{B}$ is in state $k$, the service rate is $\mu_{k}(\varepsilon)$.

Let us introduce $\varepsilon$, a small parameter of the encompassing environment of the system (including $\xi_{A}$ and $\xi_{B}$ ). The intention is to analyse the asympotic behaviour of the queueing system under the extremes of light load, when the service rates increase without limit as $\varepsilon \rightarrow 0$ (fast service), and of heavy load, when the arrival rates increase without limit as $\varepsilon \rightarrow 0$ (fast arrivals). The parameter to symbolic variables (as in $\lambda_{j}(\varepsilon)$ and $\mu_{k}(\varepsilon)$ ) is employed to emphasise that each corresponding variable may be dependent upon $\varepsilon$. 
Denote by $N_{\varepsilon}(t)$ the number of customers at service station $\Sigma_{2}$ at time $t$. Then the process $X_{\varepsilon}=\left(N_{\varepsilon}(t), \xi_{A}(t), \xi_{B}(t)\right)$ is a three-dimensional Markov chain with state space $\left\{(n, j, k): n=1, \ldots, N, j=1, \ldots, r_{A}, k=1, \ldots, r_{B}\right\}$.

The state transition rates $T\left((n, j, k),\left(n^{\prime}, l, q\right)\right)$ from state $(n, j, k)$ to state $\left(n^{\prime}, l, q\right)$ are given by

$$
\begin{aligned}
& T((n, j, k),(n, l, k))=a_{j l} \quad(j \neq l) \\
& T((n, j, k),(n, j, q))=b_{k q} \quad(k \neq q) \\
& T((n, j, k),(n+1, j, k))=\lambda_{j}(\varepsilon) \quad(n=0, \ldots, N-1) \\
& T((n, j, k),(n-1, j, k))=\mu_{k}(\varepsilon) \quad(n=1, \ldots, N) .
\end{aligned}
$$

The sojourn time, $\tau_{\varepsilon}((n, j, k))$, of $X_{\varepsilon}$ in state $(n, j, k)$, is distributed exponentially and, from the above, we see that the parameter ${ }^{2}$ of the exponential distribution is

$$
\left.\begin{array}{cl}
a_{j j}+b_{k k}+\lambda_{j}(\varepsilon) & (n=0) \\
a_{j j}+b_{k k}+\lambda_{j}(\varepsilon)+\mu_{k}(\varepsilon) & (n=1, \ldots, N-1) \\
a_{j j}+b_{k k}+\mu_{k}(\varepsilon) & (n=N)
\end{array}\right\}
$$

Therefore, the state transition probabilities are

$$
\begin{aligned}
p_{\varepsilon}((0, j, k),(0, l, k)) & =\frac{a_{j l}}{a_{j j}+b_{k k}+\lambda_{j}(\varepsilon)} \\
p_{\varepsilon}((n, j, k),(n, l, k)) & =\frac{a_{j l}}{a_{j j}+b_{k k}+\lambda_{j}(\varepsilon)+\mu_{k}(\varepsilon)} \quad(n=1, \ldots, N-1) \\
p_{\varepsilon}((N, j, k),(N, l, k)) & =\frac{a_{j l}}{a_{j j}+b_{k k}+\mu_{k}(\varepsilon)} \\
p_{\varepsilon}((0, j, k),(0, j, q)) & =\frac{b_{k q}}{a_{j j}+b_{k k}+\lambda_{j}(\varepsilon)} \\
p_{\varepsilon}((n, j, k),(n, j, q)) & =\frac{b_{k q}}{a_{j j}+b_{k k}+\lambda_{j}(\varepsilon)+\mu_{k}(\varepsilon)} \quad(n=1, \ldots, N-1) \\
p_{\varepsilon}((N, j, k),(N, j, q)) & =\frac{b_{k q}}{a_{j j}+b_{k k}+\mu_{k}(\varepsilon)} \\
p_{\varepsilon}((0, j, k),(1, j, k)) & =\frac{\lambda_{j}(\varepsilon)}{a_{j j}+b_{k k}+\lambda_{j}(\varepsilon)} \\
p_{\varepsilon}((n, j, k),(n+1, j, k)) & =\frac{\lambda_{j}(\varepsilon)}{a_{j j}+b_{k k}+\lambda_{j}(\varepsilon)+\mu_{k}(\varepsilon)} \quad(n=1, \ldots, N-1) \\
p_{\varepsilon}((n, j, k),(n-1, j, k)) & =\frac{\mu_{k}(\varepsilon)}{a_{j j}+b_{k k}+\lambda_{j}(\varepsilon)+\mu_{k}(\varepsilon)} \quad(n=1, \ldots, N-1) \\
p_{\varepsilon}((N, j, k),(N-1, j, k)) & =\frac{\mu_{k}(\varepsilon)}{a_{j j}+b_{k k}+\mu_{k}(\varepsilon)}
\end{aligned}
$$

${ }^{2}$ We follow the convention that $a_{j j}=\sum_{l \neq j} a_{j l}, b_{k k}=\sum_{q \neq k} b_{k q}$. 


\section{Asymptotic Analysis for Fast Service}

To analyse the asymptotic behaviour of the system as the service rates increase we take the $\mu_{k}(\varepsilon)$ to be

$\mu_{k}(\varepsilon)=\frac{d_{k}}{\varepsilon}$

for strictly positive ${ }^{3} d_{k}$ and the $\lambda_{j}(\varepsilon)$ to be constant (independent of $\varepsilon$ )

$\lambda_{j}(\varepsilon)=\lambda_{j}$

and let $\varepsilon \rightarrow 0$.

The transition probabilities (equation 3) then become

$$
\begin{aligned}
& p_{\varepsilon}((0, j, k),(0, l, k))=\frac{a_{j l}}{a_{j j}+b_{k k}+\lambda_{j}} \\
& p_{\varepsilon}((0, j, k),(0, j, q))=\frac{b_{k q}}{a_{j j}+b_{k k}+\lambda_{j}} \\
& p_{\varepsilon}((0, j, k),(1, j, k))=\frac{\lambda_{j}}{a_{j j}+b_{k k}+\lambda_{j}} \\
& p_{\varepsilon}((n, j, k),(n, l, k))=\varepsilon \frac{a_{j l}}{d_{k}}+o(\varepsilon) \quad(n=1, \ldots, N) \\
& p_{\varepsilon}((n, j, k),(n, j, q))=\varepsilon \frac{b_{k q}}{d_{k}}+o(\varepsilon) \quad(n=1, \ldots, N) \\
& p_{\varepsilon}((n, j, k),(n+1, j, k))=\varepsilon \frac{\lambda_{j}}{d_{k}}+o(\varepsilon) \quad(n=1, \ldots, N-1) \\
& p_{\varepsilon}((n, j, k),(n-1, j, k))=1-\varepsilon \frac{a_{j j}+b_{k k}+\lambda_{j}}{d_{k}}+o(\varepsilon) \quad(n=1, \ldots, N-1) \\
& p_{\varepsilon}((N, j, k),(N-1, j, k))=1-\varepsilon \frac{a_{j j}+b_{k k}}{d_{k}}+o(\varepsilon)
\end{aligned}
$$

Observe that the asymptotic form of the state transition probabilities suggests a partitioning of the state space into levels, as illustrated by figure 2, in which

- the probabilities of transitions from the states of each lower level to states of the next higher level are of order $\varepsilon$,

- the probabilities of transitions from the states of each higher level to states of the next lower level are of order 1 and

- the probabilities of (single step) transitions of two or more levels are identically zero.

Those features of the embedded Markov chain are requisite to application of the following theorem, due to Anisimov et al. [2] (also see [1,3,4] and references therein).

\footnotetext{
${ }^{3}$ In order to obtain the asymptotic forms of equations 4 , the possibility that any $\mu_{k}(\varepsilon) \equiv 0$ must be excluded.
} 


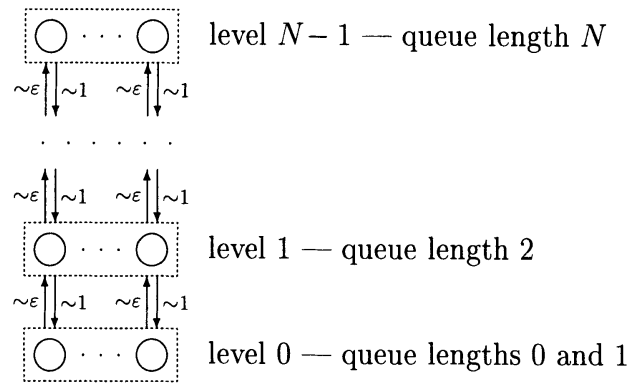

Figure 2. Typical Partitioning of State Space - Fast Service

Theorem 1 If a semi-Markov process (SMP) satisfy the conditions

1. the state space may be partitioned into "levels" $S_{0}, S_{1}, \ldots, S_{m+1}$ such that the probabilities $p_{\varepsilon}(j, k)$ of transition from state $j$ to state $k$ of the embedded Markov chain satisfy

(a) $p_{\varepsilon}(j, k) \rightarrow p_{0}(j, k)$ as $\varepsilon \rightarrow 0 \forall j, k \in S_{0}$,

(b) $p_{\varepsilon}(j, k)=\varepsilon \alpha_{l}(j, k)+o(\varepsilon) \forall j \in S_{l}, \forall k \in S_{l+1}, l=0, \ldots, m$,

(c) $p_{\varepsilon}(j, k) \rightarrow 0$ as $\varepsilon \rightarrow 0 \forall j, k \in S_{l}, l=1, \ldots, m$,

(d) $p_{\varepsilon}(j, k) \equiv 0 \forall j \in S_{l}, \forall k \in S_{q},|q-k|>1$

2. the sojourn $\tau_{\varepsilon}(j, k)$ of the SMP in state $j$ given the next state be $k$ be such that its characteristic function $\varphi_{\varepsilon}(u, j, k)=\mathrm{E}\left[\exp \left(i u \tau_{\varepsilon}(j, k)\right)\right]$ satisfy ${ }^{4}$

$\varphi_{\varepsilon}\left(\beta_{\varepsilon} u, j, k\right)=1+\chi_{j k}(u) \varepsilon^{m+1}+o\left(\varepsilon^{m+1}\right)$

where $\beta_{\varepsilon}$ be a normalising constant

then the characteristic function $\psi_{\varepsilon}(u, j)$ of the sojourn in the subset $\langle\alpha\rangle=\bigcup_{l=0}^{m} S_{l}$ starting from state $j \in\langle\alpha\rangle$, normalised by $\beta_{\varepsilon}$, converges to the limit

$\lim _{\varepsilon \rightarrow 0} \psi_{\varepsilon}\left(\beta_{\varepsilon} u, j\right)=\frac{1}{1-A(u)}$,

independent of the initial state $j$, where

$A(u)=\frac{\sum_{k, j \in S_{0}} \pi_{0}(k) p_{0}(k, j) \chi_{k j}(u)}{\sum \pi_{0}\left(k_{0}\right) \alpha_{0}\left(k_{0}, k_{1}\right) \ldots \alpha_{m}\left(k_{m}, k_{m+1}\right)}$,

${ }^{4}$ Here $i$ is the imaginary number $\sqrt{-1}$ 
(where the sum in the denominator is over all $k_{0} \in S_{0}, \ldots, k_{m+1} \in S_{m+1}$ ) and $\pi_{0}(k)$ is the stationary probability for the embedded Markov chain to be in state $k \in S_{0}$ in the limit when $\varepsilon=0$.

In particular, if $\chi_{j k}(u)$ is of the form $i x_{j k} u$ (where $i=\sqrt{-1}$ ), the limiting distribution is exponential with parameter

$$
\frac{\sum \pi_{0}\left(k_{0}\right) \alpha_{0}\left(k_{0}, k_{1}\right) \ldots \alpha_{m}\left(k_{m}, k_{m+1}\right)}{\sum_{k, j \in S_{0}} \pi_{0}(k) p_{0}(k, j) x_{k j}}
$$

(where the sum in the numerator is over all $k_{0} \in S_{0}, \ldots, k_{m+1} \in S_{m+1}$ ).

- From equations 4 , it is seen that condition 1 for theorem 1 is satisfied with

$$
\begin{aligned}
& S_{0}=\{(0, j, k),(1, j, k)\}, S_{n}=\{(n+1, j, k)\} \\
& j=1, \ldots, r_{A}, k=1, \ldots, r_{B}, n=1, \ldots, N-1 .
\end{aligned}
$$

- Now, the sojourn of $X_{\varepsilon}$ in state $(n, j, k)$ is independent of the next state of $X_{\varepsilon}$ and is distributed exponentially, with parameter $\lambda$ (say) as given by the appropriate line at (2) above, and its characteristic function is

$$
\varphi\left(u,(n, j, k),\left(n^{\prime}, l, q\right)\right)=\frac{\lambda}{\lambda-i u} .
$$

Consequently,

$$
\varphi\left(\varepsilon^{m+1} u,(n, j, k),\left(n^{\prime}, l, q\right)\right)=\frac{\lambda}{\lambda-i \varepsilon^{m+1} u}=1+i \varepsilon^{m+1} \frac{u}{\lambda}+o\left(\varepsilon^{m+1}\right)
$$

and so condition 2 of theorem 1 is satisfied with $\beta_{\varepsilon}=\varepsilon^{m+1}$ and

$$
x_{(n, j, k),\left(n^{\prime}, l, q\right)}= \begin{cases}\frac{1}{a_{j j}+b_{k k}+\lambda_{j}} & (n=0) \\ 0 & (n=1, \ldots, N)\end{cases}
$$

- The limiting values for the transition probabilities (i.e. for $\varepsilon=0$ in equations 4 ) imply that $\pi_{0}$ satisfies

$$
\begin{aligned}
& \pi_{0}(0, l, q)=\sum_{j \neq l} \pi_{0}(0, j, q) \frac{a_{j l}}{a_{j j}+b_{q q}+\lambda_{j}}+\sum_{k \neq q} \pi_{0}(0, l, k) \frac{b_{k q}}{a_{l l}+b_{k k}+\lambda_{l}}+\pi_{0}(1, l, q), \\
& \pi_{0}(1, l, q)=\pi_{0}(0, l, q) \frac{\lambda_{l}}{a_{l l}+b_{q q}+\lambda_{l}}
\end{aligned}
$$

which, together with $\pi_{A}(l) a_{l l}=\sum_{j \neq l} \pi_{A}(j) a_{j l}$ and $\pi_{B}(q) b_{q q}=\sum_{k \neq q} \pi_{B}(k) b_{k q}$, yield

$$
\pi_{0}(n, j, k)=\left\{\begin{array}{cc}
\frac{1}{Z} \pi_{A}(j) \pi_{B}(k)\left(a_{j j}+b_{k k}+\lambda_{j}\right) & (n=0) \\
\frac{1}{Z} \pi_{A}(j) \pi_{B}(k) \lambda_{j} & (n=1)
\end{array}\right.
$$


$j=1, \ldots, r_{A}, k=1, \ldots, r_{B}$ where $Z$ is the normalising constant

$$
Z=\sum_{l=1}^{r_{A}} \sum_{q=1}^{r_{B}} \pi_{A}(l) \pi_{B}(q)\left(a_{l l}+b_{q q}+2 \lambda_{l}\right)
$$

Write $\Omega_{\varepsilon}(m)$ for the random variable of the sojourn of the system in levels 0 to $m$ (i.e. the random variable for which $\psi_{\varepsilon}\left(\beta_{\varepsilon} u, j\right)$ is the characteristic function). Theorem 1 may be applied to show that the normalised sojourn $\varepsilon^{m+1} \Omega_{\varepsilon}(m)$ of the system in levels 0 to $m$ (queue lengths 0 to $m+1$ ) converges weakly to an exponential distribution with parameter

$\Lambda_{m}=\sum_{j=1}^{r_{A}} \sum_{k=1}^{r_{B}} \pi_{A}(j) \pi_{B}(k) \frac{\lambda_{j}^{m+2}}{d_{k}^{m+1}}=\sum_{j=1}^{r_{A}} \pi_{A}(j) \lambda_{j}^{m+2} \sum_{k=1}^{r_{B}} \frac{\pi_{B}(k)}{d_{k}^{m+1}}$,

$m=0, \ldots, N-2$. Then

$\operatorname{Pr}\left[\Omega_{\varepsilon}(m) \leq t\right]=\operatorname{Pr}\left[\varepsilon^{m+1} \Omega_{\varepsilon}(m) \leq \varepsilon^{m+1} t\right] \approx 1-\exp \left(-\varepsilon^{m+1} \Lambda_{m} t\right)$

and, recalling that $d_{k}=\varepsilon \mu_{k}(\varepsilon)$, it is concluded that the sojourn of the system in levels 0 to $m$ converges weakly to an exponential distribution with parameter

$\varepsilon^{m+1} \Lambda_{m}=\sum_{j=1}^{r_{A}} \pi_{A}(j) \lambda_{j}^{m+2} \sum_{k=1}^{r_{B}} \frac{\pi_{B}(k)}{\mu_{k}(\varepsilon)^{m+1}}$

as $\varepsilon \rightarrow 0$ for all $m, m=0, \ldots, N-2$.

\section{Asymptotic Analysis for Fast Arrivals}

To analyse the asymptotic behaviour of the system as the arrival rates increase take the $\lambda_{j}(\varepsilon)$ to be

$\lambda_{j}(\varepsilon)=\frac{c_{j}}{\varepsilon}$

for strictly positive ${ }^{5} c_{j}$ and the $\mu_{k}(\varepsilon)$ to be constant (independent of $\varepsilon$ )

$\mu_{k}(\varepsilon)=\mu_{k}$

and let $\varepsilon \rightarrow 0$.

Then, proceeding in a way similar to that of the previous section for fast service,

${ }^{5}$ In order to obtain the asymptotic forms of equations 6 , the possibility that any $\lambda_{j}(\varepsilon) \equiv 0$ must be excluded. 
- use the asymptotic form

$$
\begin{aligned}
& p_{\varepsilon}((n, j, k),(n, l, k))=\varepsilon \frac{a_{j l}}{c_{j}}+o(\varepsilon) \\
& (n=0, \ldots, N-1) \\
& p_{\varepsilon}((n, j, k),(n, j, q))=\varepsilon \frac{b_{k q}}{c_{j}}+o(\varepsilon) \quad(n=0, \ldots, N-1) \\
& p_{\varepsilon}((0, j, k),(1, j, q))=1-\varepsilon \frac{a_{j j}+b_{k k}}{c_{j}}+o(\varepsilon) \\
& p_{\varepsilon}((n, j, k),(n+1, j, k))=1-\varepsilon \frac{a_{j j}+b_{k k}+\mu_{k}}{c_{j}}+o(\varepsilon) \quad(n=1, \ldots, N-1) \\
& p_{\varepsilon}((n, j, k),(n-1, j, k))=\varepsilon \frac{\mu_{k}}{c_{j}}+o(\varepsilon) \quad(n=1, \ldots, N-1) \\
& p_{\varepsilon}((N, j, k),(N-1, j, k))=\frac{\mu_{k}}{a_{j j}+b_{k k}+\mu_{k}}+o(\varepsilon) \\
& p_{\varepsilon}((N, j, k),(N, l, k))=\frac{a_{j l}}{a_{j j}+b_{k k}+\mu_{k}}+o(\varepsilon) \\
& p_{\varepsilon}((N, j, k),(N, j, q))=\frac{b_{k q}}{a_{j j}+b_{k k}+\mu_{k}}+o(\varepsilon)
\end{aligned}
$$

of the transition probabilities,

- identify the levels

$$
\begin{aligned}
& S_{0}=\{(N, j, k),(N-1, j, k)\}, S_{l}=\{(N-1-l, j, k)\} \\
& j=1, \ldots, r_{A}, k=1, \ldots, r_{B}, l=1, \ldots, N-1,
\end{aligned}
$$

- use form(2) to see that condition 2 of theorem 1 is satisfied with $\beta_{\varepsilon}=\varepsilon^{m+1}$ and

$$
x_{(n, j, k),\left(n^{\prime}, l, q\right)}= \begin{cases}0 & (n=0, \ldots, N-1) \\ \frac{1}{a_{j j}+b_{k k}+\mu_{k}} & (n=N)\end{cases}
$$

- use the limiting values for the transition probabilities (i.e. for $\varepsilon=0$ in equations 6 ) to obtain $\pi_{0}$ as

$$
\pi_{0}(n, j, k)=\left\{\begin{array}{cl}
\frac{1}{Z} \pi_{A}(j) \pi_{B}(k)\left(a_{j j}+b_{k k}+\mu_{k}\right) & (n=N) \\
\frac{1}{Z} \pi_{A}(j) \pi_{B}(k) \mu_{k} & (n=N-1)
\end{array}\right.
$$

$j=1, \ldots, r_{A}, k=1, \ldots, r_{B}$, where $Z$ is the normalising constant

$$
Z=\sum_{l=1}^{r_{A}} \sum_{q=1}^{r_{B}} \pi_{A}(l) \pi_{B}(q)\left(a_{l l}+b_{q q}+2 \mu_{q}\right)
$$


- apply theorem 1 to show that the sojourn $\Omega_{\varepsilon}(m)$ of the system in levels 0 to $m$ (queue lengths $N-1-m$ to $N$ ) converges weakly to an exponential distribution with parameter

$$
\varepsilon^{m+1} \Lambda_{m}=\sum_{j=1}^{r_{A}} \frac{\pi_{A}(j)}{\lambda_{j}(\varepsilon)^{m+1}} \sum_{k=1}^{r_{B}} \pi_{B}(k) \mu_{k}^{m+2}
$$

as $\varepsilon \rightarrow 0$ for all $m, m=0, \ldots, N-2$.

The same results may also be obtained from the results of the preceeding section by observing that fast arrivals to server $\Sigma_{1}$ are fast service at server $\Sigma_{2}$ and interchanging the variables associated with $\Sigma_{1}$ and $\Sigma_{2}$, i.e. by exchanging $\lambda_{j}, \pi_{A}(j)$, etc. with $\mu_{k}, \pi_{B}(k)$, etc. and by replacing $n$ by $N-n$ in the results for fast service.

Observe also that $\Omega_{\varepsilon}(N-2)$, the sojourn in states with queue lengths 1 to $N$, is the busy period.

\section{Location of Boundaries}

Having determined asymptotic behaviour of MMPP/MMPP/1/N systems under fast service and fast arrivals, it is no longer necessary to consider the effect of variation in $\varepsilon$. In this and subsequent sections notation for $\lambda_{j}(\varepsilon)$ and $\mu_{k}(\varepsilon)$ is simplified to $\lambda_{j}$ and $\mu_{k}$.

Purely as an expedient, the simplifying assumption is made that utilisation $U$ may be approximated by a renewal result

$U=\frac{\text { busy period }}{\text { idle period }+ \text { busy period }}$.

It turns out that this crude approximation readily yields the boundaries between those regions in which correlation is not significant and the region in which correlation is significant. The term "B-approximation" is introduced in order to emphasise that the expressions resulting from such gross simplification are used only to locate the boundaries and are not, themselves, expected to be useful approximations to utilisation, etc. More accurate asymptotic approximations to utilisation may be obtained (see Appendix A) and may be employed in the defined regions but are not so convenient to determination of those regions.

In this section, first asymptotic B-approximations to utilisation and to throughput are derived under the assumption of fast arrivals and the simplifying assumption. Then, using the dual model, fast service asymptotic B-approximations to throughput and to utilisation are derived from the fast arrivals B-approximations. Finally, the B-approximations to utilisation are expressed in terms of traffic intensity - which leads to simple closed form expressions for the locations of the boundaries of the low intensity and high intensity regions in which correlation has no significant effect on system behaviour.

\subsection{Fast Arrivals}

From the previous section, the fast arrivals approximation to the mean busy period is

$$
\frac{1}{\varepsilon^{N-1} \Lambda_{N-2}}=\frac{1}{\sum_{j=1}^{r_{A}} \frac{\pi_{A}(j)}{\lambda_{j}^{N-1}} \sum_{k=1}^{r_{B}} \pi_{B}(k) \mu_{k}^{N}} .
$$


It is readily seen that the mean idle period is the mean interarrival period and that the mean arrival rate is given by

$\sum_{j=1}^{r_{A}} \pi_{A}(j) \lambda_{j}$

Therefore, the fast arrivals B-approximation to utilisation, $U_{a}$, may be taken to be

$U_{a} \approx \frac{\sum_{j=1}^{r_{A}} \pi_{A}(j) \lambda_{j}}{\sum_{j=1}^{r_{A}} \pi_{A}(j) \lambda_{j}+\sum_{j=1}^{r_{A}} \frac{\pi_{A}(j)}{\lambda_{j}^{N-1}} \sum_{k=1}^{r_{B}} \pi_{B}(k) \mu_{k}^{N}}$.

Throughput is utilisation multiplied by mean service rate, i.e.

$$
\frac{\sum_{j=1}^{r_{A}} \pi_{A}(j) \lambda_{j} \sum_{k=1}^{r_{B}} \pi_{B}(k) \mu_{k}}{\sum_{j=1}^{r_{A}} \pi_{A}(j) \lambda_{j}+\sum_{j=1}^{r_{A}} \frac{\pi_{A}(j)}{\lambda_{j}^{N-1}} \sum_{k=1}^{r_{B}} \pi_{B}(k) \mu_{k}^{N}} .
$$

\subsection{Fast Service}

Because fast arrivals to server $\Sigma_{2}$ is fast service at $\Sigma_{1}$ and because throughput at $\Sigma_{1}$ must equal throughput at $\Sigma_{2}$, it is immediately obvious (by exchanging variables) that the fast service B-approximation to throughput is

$$
\frac{\sum_{j=1}^{r_{A}} \pi_{A}(j) \lambda_{j} \sum_{k=1}^{r_{B}} \pi_{B}(k) \mu_{k}}{\sum_{k=1}^{r_{B}} \pi_{B}(k) \mu_{k}+\sum_{j=1}^{r_{A}} \pi_{A}(j) \lambda_{j}^{N} \sum_{k=1}^{r_{B}} \frac{\pi_{B}(k)}{\mu_{k}^{N-1}}} .
$$

Then, dividing throughput by mean service rate

$$
\sum_{k=1}^{r_{B}} \pi_{B}(k) \mu_{k}
$$

gives the fast service B-approximation to utilisation

$$
U_{s} \approx \frac{\sum_{j=1}^{r_{A}} \pi_{A}(j) \lambda_{j}}{\sum_{k=1}^{r_{B}} \pi_{B}(k) \mu_{k}+\sum_{j=1}^{r_{A}} \pi_{A}(j) \lambda_{j}^{N} \sum_{k=1}^{r_{B}} \frac{\pi_{B}(k)}{\mu_{k}^{N-1}}} .
$$

\subsection{Boundaries of the Low Intensity and High Intensity Regions}

For fast arrivals, the B-approximation to utilisation is given by equation 8 . This approximation is expected to be good for high intensity (large values of the $\lambda_{j}$ ) but the objective is to locate the lower limit of intensity for which the approximation remains good. Consideration of the second term of the denominator shows that, until the intensity is so high that the least among the $\lambda_{j}$ exceeds the greatest of the $\mu_{k}$, this second term 
of the denominator will be greater than unity and will increase with $N$, i.e. $U_{a} \rightarrow 0$ as $N \rightarrow \infty$ while ever the intensity be sufficiently low.

Next, consider how $U_{a}$ varies with intensity when the $\lambda_{j}$ are varied proportionally, i.e. chose the $\lambda_{j}$ corresponding to some arbitrary intensity, then scale the $\lambda_{j}$ by applying the same multiplier to each $\lambda_{j}$ to produce a different intensity. For any initial values of the $\lambda_{j}$, the corresponding initial value of intensity is $\bar{\lambda} / \bar{\mu}$ where $\bar{\lambda}$ is the mean arrival rate

$\bar{\lambda}=\sum_{j=1}^{r_{A}} \pi_{A}(j) \lambda_{j}$

and $\bar{\mu}$ is the mean service rate

$\bar{\mu}=\sum_{k=1}^{r_{B}} \pi_{B}(k) \mu_{k}$.

It is obvious that, to achieve a given value of intensity (say) $\rho$, each of the initial values of the $\lambda_{j}$ should be multiplied by

$\rho \bar{\mu} / \bar{\lambda}$.

Equation 8 then becomes

$U_{a} \approx \frac{\sum_{j=1}^{r_{A}} \pi_{A}(j) \lambda_{j}\left(\frac{\rho \bar{\mu}}{\bar{\lambda}}\right)}{\sum_{j=1}^{r_{A}} \pi_{A}(j) \lambda_{j}\left(\frac{\rho \bar{\mu}}{\bar{\lambda}}\right)+\sum_{j=1}^{r_{A}} \frac{\pi_{A}(j)}{\lambda_{j}^{N-1}}\left(\frac{\bar{\lambda}}{\rho \bar{\mu}}\right)^{N-1} \sum_{k=1}^{r_{B}} \pi_{B}(k) \mu_{k}^{N}}$

which may be expressed as

$U_{a} \approx \frac{\rho^{N}}{X_{a}^{N}+\rho^{N}}$

where

$X_{a}^{N}=\sum_{j=1}^{r_{A}} \frac{\pi_{A}(j)}{\lambda_{j}^{N-1}} \sum_{k=1}^{r_{B}} \pi_{B}(k) \mu_{k}^{N} \frac{\bar{\lambda}^{N-1}}{\bar{\mu}^{N}}=\sum_{j=1}^{r_{A}} \frac{\pi_{A}(j)}{\left(\lambda_{j} / \bar{\lambda}\right)^{N-1}} \sum_{k=1}^{r_{B}} \pi_{B}(k)\left(\mu_{k} / \bar{\mu}\right)^{N}$.

Observe that

- the same result is obtained when varying the $\mu_{k}$ proportionally (the multiplier is $\bar{\lambda} /(\rho \bar{\mu})$,

- the value $X_{a}^{N}$ depends on the relative values of the $\lambda_{j}$ and of the $\mu_{k}$ rather than on the absolute values $-X_{a}^{N}$ is independent of the value of the reference intensity,

- the B-approximation is essentially independent of the degree of correlation between successive interarrival periods or between service periods - for all processes with different degrees of correlation but sharing the same stationary distribution of environmental phases the same fast arrivals asymptotic B-approximation results, 
- except for the trivial case of an unchanging environment (or, equivalently, when the $\lambda_{j}$ are all identical and the $\mu_{k}$ are all identical) $X_{a}^{N}$ is strictly greater than 1 and increases with $N$,

- with increasing $N$, the value of $X_{a}^{N}$ is increasingly dominated by the relative magnitude of the fastest service rate and the slowest arrival rate and tends to a finite limit

$$
X_{a} \triangleq \lim _{N \rightarrow \infty} \sqrt[N]{X_{a}^{N}}=\frac{\max _{k}\left(\mu_{k}\right) / \bar{\mu}}{\min _{j}\left(\lambda_{j}\right) / \bar{\lambda}} .
$$

It is clear that the magnitude of $X_{a}^{N}$ is directly related to the range of validity of the fast arrivals B-approximation to utilisation - a higher value for $X_{a}^{N}$ implies a reduced range of validity.

For fast service, similar conclusions may be reached. Considering the expression 9 for the fast service B-approximation to utilisation, we see that, when the intensity is so high that the least among the $\mu_{k}$ exceeds the greatest of the $\lambda_{j}$, this second term of the denominator will be greater than unity and will increase with $N$, i.e. $U_{s} \rightarrow 0$ as $N \rightarrow \infty$ while ever the intensity be sufficiently high. Equation 9 may be written

$U_{s} \approx \frac{\rho}{1+X_{s}^{N} \rho^{N}}$

where

$X_{s}^{N}=\sum_{j=1}^{r_{A}} \pi_{A}(j)\left(\lambda_{j} / \bar{\lambda}\right)^{N} \sum_{k=1}^{r_{B}} \frac{\pi_{B}(k)}{\left(\mu_{k} / \bar{\mu}\right)^{N-1}}$.

A higher value for $X_{s}^{N}$ implies a reduced range of validity for the fast service B-approximation to utilisation and

$X_{s} \triangleq \lim _{N \rightarrow \infty} \sqrt[N]{X_{s}^{N}}=\frac{\max _{j}\left(\lambda_{j}\right) / \bar{\lambda}}{\min _{k}\left(\mu_{k}\right) / \bar{\mu}}$.

In summary, higher values for $X_{a}^{N}$ and $X_{s}^{N}$ imply reduced ranges of validity for the asymptotic B-approximations to utilisation. The magnitudes of $X_{a}^{N}$ and $X_{s}^{N}$ are dominated (with increasing $N$ ) only by the spread of arrival and service rates: rates of intermediate magnitude in other phases are of reducing effect.

Finally, it is clear from equations 10 and 12 that, with increasing intensity, $U_{s}$ adheres closely to the $\mathrm{M} / \mathrm{M} / 1 / \mathrm{N}$ utilisation/intensity curve until $\rho$ approaches $1 / \sqrt[N]{X_{s}^{N}}$ and that $U_{a}$ conforms to the $\mathrm{M} / \mathrm{M} / 1 / \mathrm{N}$ utilisation/intensity curve only when $\rho$ exceeds $\sqrt[N]{X_{a}^{N}}$. The position of the boundaries may be determined to be those values of intensity at which, for some tolerence $\delta$, the B-approximation differs from the utilisation $U$ of the $\mathrm{M} / \mathrm{M} / 1 / \mathrm{N}$ system by $U \delta$. Thus the boundary $\rho=L$ to the low intensity region is given by that value of intensity for which $\left|\frac{U-U_{s}}{U}\right|=\delta$, i.e.

$1-\frac{1}{1+X_{s}^{N} L^{N}} \frac{1-L^{N+1}}{1-L^{N}}=\delta$ 
Observe that the term $\frac{1-L^{N+1}}{1-L^{N}}$ is within $.5 \times 10^{-3}$ of unity whenever $0 \leq L \leq 0.5$ and $N \geq 10$. So, for all practical purposes,

$L \approx\left(\frac{\delta}{1-\delta} \frac{1}{X_{s}^{N}}\right)^{\frac{1}{N}}$

Similarly, the location $\rho=H$ of the boundary to the high intensity region may be approximated by

$H \approx\left(\frac{1-\delta}{\delta} X_{a}^{N}\right)^{\frac{1}{N}}$

For small $\delta$ and large $N$, the bounds on $\rho$ given by expressions 14 and 15 are not sensitive to the exact value chosen for $\delta$ and so define the region boundaries quite precisely. However, for smaller $N$, the magnitude of the term $\left(\frac{\delta}{1-\delta}\right)^{\frac{1}{N}}$, which appears both in equation 14 and in equation 15, is relatively sensitive to the choice of $\delta$. Therefore, for conservative estimates of the ranges in which approximations be valid, it is appropriate to chose a small value (say $1 \%$ ) for $\delta$.

The numerical results which are presented in the next section indicate that those ranges of intensity do indeed coincide with the ranges for which correlation has little effect on system behaviour.

\section{Results}

We examine the fast arrivals and fast service asymptotic B-approximations to utilisation first in a stable environment and then in a randomly changing environment.

\section{1. $M / M / 1 / N$}

In a stable environment, the model system reduces to the familiar $M / M / 1 / N$ queueing system. The renewal result for utilisation is valid and so the B-approximations should give good results.

The fast arrivals and fast service asymptotic approximations to utilisation reduce to the expressions given below, in which the exact value is also given (for ease of comparison).

$U=\left\{\begin{array}{cl}\frac{\rho}{1+\rho^{N}} & \text { fast service } \\ \frac{\rho^{N}}{1+\rho^{N}} & \text { fast arrivals } \\ \frac{\rho\left(1-\rho^{N}\right)}{1-\rho^{N+1}} & \text { exact }\end{array}\right.$

The general behaviour of the asymptotic approximations is shown by the graphs of figure 3 .

The fast service approximation is good for small values of $\rho$. If the relative error tolerance is $\delta$, the fast service approximation is good for $\rho \in[0, \sqrt[N+1]{\delta})$. E.g., with $\delta=1 \%$, the fast service approximation is good for $\rho$ up to $80 \%$ with $N=20$ and, for $N=40$, up to $89 \%$. As $N \rightarrow \infty$ the approximation becomes exact for $\rho \in[0,1)$. 

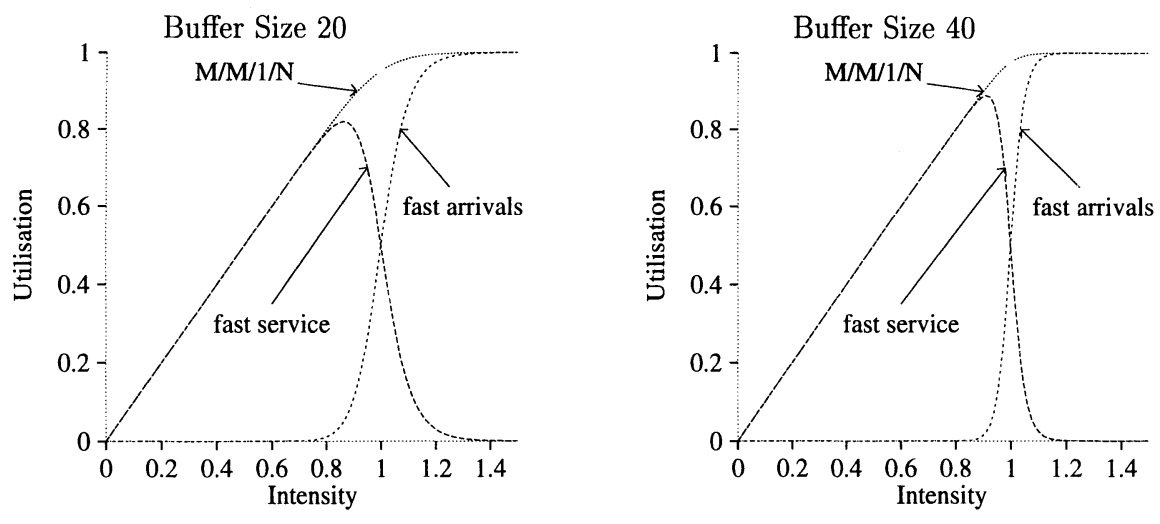

Figure 3. Comparison of fast service and fast arrivals asymptotic approximations for utilisation against exact results for $\mathrm{M} / \mathrm{M} / 1 / \mathrm{N}$ system.

The fast arrivals approximation is good for large values of $\rho$. If the relative error tolerance is $\delta$, the fast arrivals approximation is good for $\rho \in(1 / \sqrt[N+1]{\delta}, \infty)$. E.g., with $\delta=1 \%$, the fast arrivals approximation is good for $\rho$ greater than 1.25 with $N=20$ and, for $N=40$, greater than 1.12. As $N \rightarrow \infty$ the approximation becomes exact for $\rho \in(1, \infty)$.

\subsection{MMPP/MMPP/1/N with 2-phase Arrivals and Service Processes}

From equation 13 we see that, for low intensities, the behaviour of the fast service B-approximation $U_{s}$ with increasing intensity is similar to that in the $\mathrm{M} / \mathrm{M} / 1 / \mathrm{N}$ case until $X_{s}^{N} \rho^{N}$ becomes significant. Similarly, from equation 11, the behaviour of the fast arrivals $\mathrm{B}$-approximation $U_{a}$ with decreasing intensity (from high intensity) is similar to that in the $\mathrm{M} / \mathrm{M} / 1 / \mathrm{N}$ case - until $X_{a}^{N} / \rho^{N}$ becomes significant.

Figures 4, 5 show graphs of the asymptotic B-approximations against exact results for various parameters of an MMPP/MMPP $/ 1 / \mathrm{N}$ system. The exact results are given for three models (lines labelled 1,2 and 3) relate to processes with substantially different phase change rates (giving different degrees of correlation between succeeding events, indices of dispersion, etc.) but with the same probability distribution for the phase of the Markov chain modulating the Poisson process and the same relative arrival and service rates in each phase. These latter features are just those which the exact models share with the asymptotic B-approximations.

In chosing parameters for the examples, the intention was to impose a severe test of the asymptotic approximation method in conditions unfavourable to the accuracy of the method. In each example

- the phase change rates are varied by a factor of 10,000 ,

- the buffer sizes are relatively small: the analysis indicates that smaller buffer size leads to less precise definition of region boundaries, 

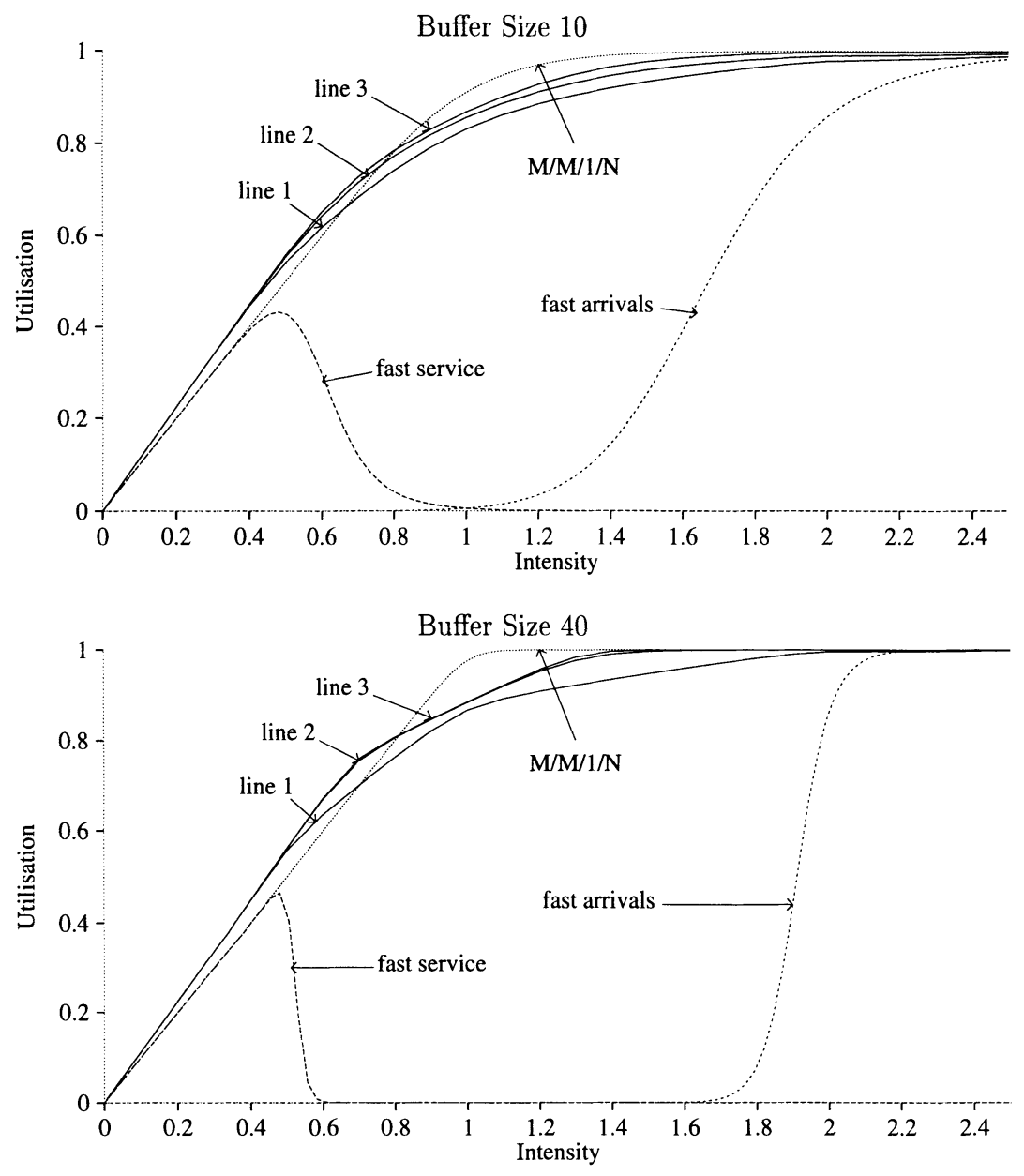

Figure 4. Comparison of fast service and fast arrivals B-approximations for utilisation against exact results for a family of MMPP/MMPP/1/N systems with buffer sizes $N=10$ and $N=40$. The family is $\pi_{A}(1)=.4985, \pi_{A}(2)=.5015, \lambda_{1} / \lambda_{2}=2 / 1, \pi_{B}(1)=.499$, $\pi_{B}(2)=.501, \mu_{1} / \mu_{2}=2 / 1$.

\begin{tabular}{|c|ccc|c|c|c|c|}
\hline$\lambda_{1} / \lambda_{2}$ & line & $\sigma_{1}$ & $\sigma_{2}$ & $\mu_{1}$ & $\mu_{2}$ & $\tau_{1}$ & $\tau_{2}$ \\
\hline \multirow{3}{*}{$2 / 1$} & 1 & .001003 & .000997 & & & & \\
& 2 & .1003 & .0997 & 1.4 & .7 & .001002 & .000998 \\
& 3 & 10.03 & 9.97 & & & & \\
\hline
\end{tabular}

For tolerance $\delta=.01$, the region boundaries are $L \approx .36, H \approx 2.7$ for $N=10$ and $L \approx .47, H \approx 2.14$ for $N=40$ and $L \rightarrow .5, H \rightarrow 2$ as $N \rightarrow \infty$. 

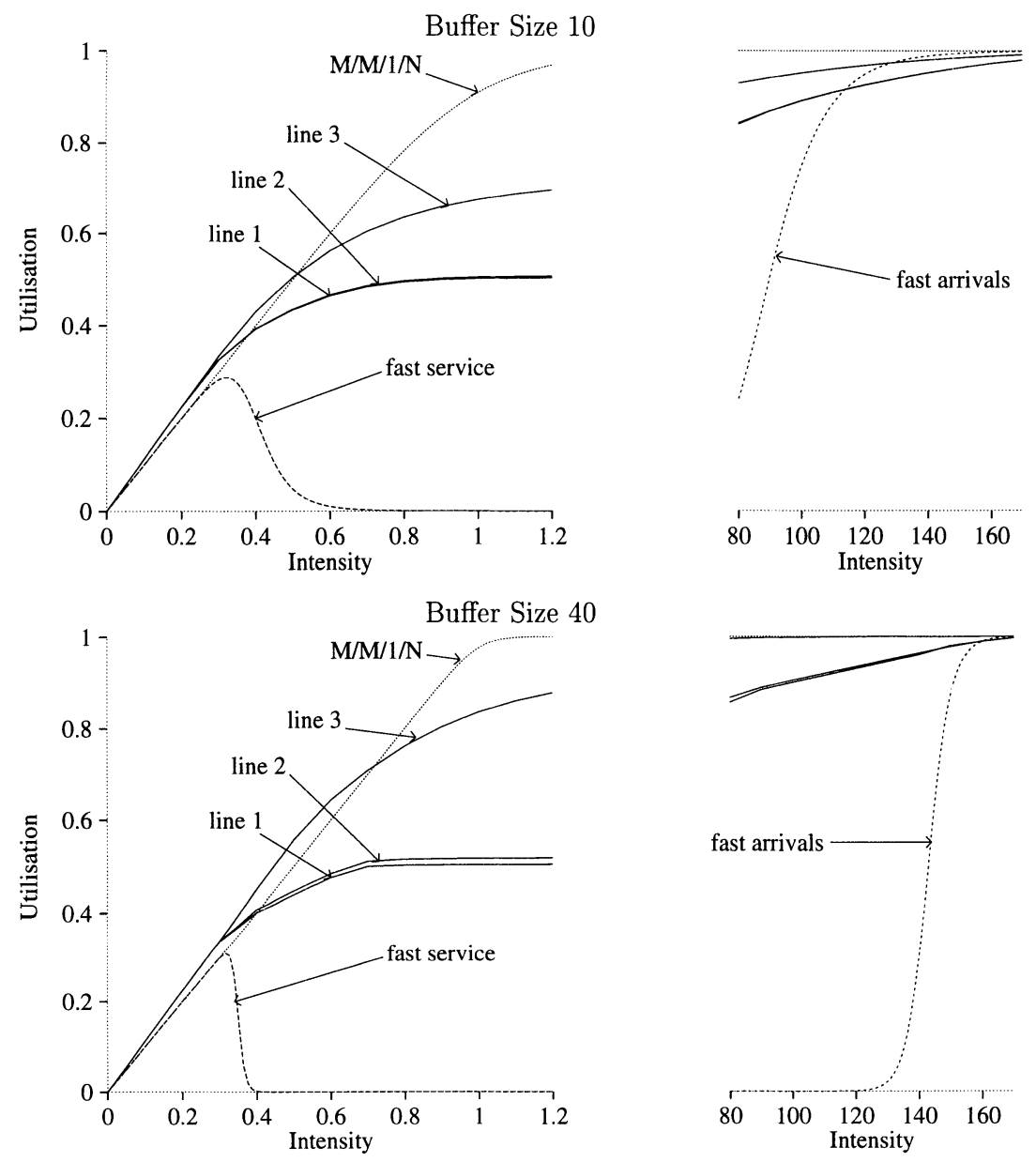

Figure 5. Comparison of fast service and fast arrivals B-approximations for utilisation against exact results for for a family of MMPP/MMPP/1/N systems with buffer sizes $N=10$ and $N=40$. The family is $\pi_{A}(1)=.4985, \pi_{A}(2)=.5015, \lambda_{1} / \lambda_{2}=250 / 1$, $\pi_{B}(1)=.499, \pi_{B}(2)=.501, \mu_{1} / \mu_{2}=2 / 1$.

\begin{tabular}{|c|cll|c|c|c|c|}
\hline$\lambda_{1} / \lambda_{2}$ & line & $\sigma_{1}$ & $\sigma_{2}$ & $\mu_{1}$ & $\mu_{2}$ & $\tau_{1}$ & $\tau_{2}$ \\
\hline \multirow{2}{*}{$250 / 1$} & 1 & .001003 & .000997 & & & & \\
& 2 & .1003 & .0997 & 200 & 100 & .001002 & .000998 \\
& 3 & 10.03 & 9.97 & & & & \\
\hline
\end{tabular}

For tolerance $\delta=.01$, the region boundaries are $L \approx .25, H \approx 179$ for $N=10$ and $L \approx .31, H \approx 170$ for $N=40$ and $L \rightarrow .334, H \rightarrow 167$ as $N \rightarrow \infty$. 
- the arrival and service MMPP's each have just two phases: the analysis shows the dominant effect of only the fastest and the slowest amongst the arrival rates and amongst the service rates: phases with intermediate rates would, at most, ameliorate the extremes of system behaviour.

The two examples serve to illustrate the effect of different spread between arrival rates: in the first example (figure 4 the spread is a factor of 2 whereas in the second example (figure 5 the spread is a factor of 250 .

The notations $\sigma_{1}$ and $\sigma_{2}$ have been introduced for the phase change rates of the arrivals process and $\tau_{1}$ and $\tau_{2}$ for those of the service process (i.e. $a_{11}=a_{12}=\sigma_{1}, a_{21}=a_{22}=\sigma_{2}$, $b_{11}=b_{12}=\tau_{1}, b_{21}=b_{22}=\tau_{2}$ ).

In figures 4,5 the exact graphs, resulting from numerical computation, were obtained by varying $\lambda_{1}, \lambda_{2}$ for various values of $\sigma_{1}, \sigma_{2}$ whilst keeping $\mu_{1}, \mu_{2}, \tau_{1}$ and $\tau_{2}$ fixed. Similar results were obtained by varying $\mu_{1}, \mu_{2}$ for various values of $\tau_{1}, \tau_{2}$ whilst keeping $\lambda_{1}, \lambda_{2}$, $\sigma_{1}$ and $\sigma_{2}$ fixed - as is to be expected from the analysis: such graphs are not included here because they would not add any further information.

The exact results indicate that MMPP/MMPP/1/N systems are composed of families. The characteristic of each family is that all member systems have the same steady state distributions of arrivals phases and the same steady state distributions of service phases. It is seen that

1. all members of each family of MMPP/MMPP/1/N systems have the same low intensity region and the same high intensity region - even though the phase transition rates (and correlation) vary enormously between different members,

2. in the region of intermediate intensity the results for different members diverge rapidly at the region boundaries and, thus, the region boundaries are given quite precisely,

3. the location of the region boundaries are given as simple closed form expressions in the model parameters - phase distribution and relative rates of arrival and service MMPP's and buffer size.

The authors are not aware of any explanation as to why, for all members of a family, the graphs should separate from the same point. However, given that such points exist, it is intuitively obvious that the same feature should be exhibited in the asymptotic B-approximations derived above: the upper boundary of the low intensity region can be associated with the point at which the graph of the fast service B-approximation diverges from that of the $M / M / 1 / N$; the lower boundary of the high intensity region can be associated with the point at which the graph of the fast arrivals B-approximation approaches that of the $\mathrm{M} / \mathrm{M} / 1 / \mathrm{N}$.

As expected, from the analysis above, the graphs of fast service and fast arrivals asymptotic B-approximations for utilisation conform to that of the $\mathrm{M} / \mathrm{M} / 1 / \mathrm{N}$ system, within limited ranges, but, unlike those for a stable environment, the ranges of validity are not increased by increasing $N$ but are restricted (by $X_{s}^{N}>1, X_{a}^{N}>1$ ).

The suggestion is

1. that, in the low intensity and high intensity regions, the behaviour of the system is least dependent upon the parameters chosen and, in the region of intermediate intensity, 
the behaviour of the system is more sensitive to choice of parameters of the Markov modulated processes,

2. that the regions are quite distinct and have definite boundaries,

3. that the boundaries are determined by the factors $X_{s}^{N}$ and $X_{a}^{N}$, as shown by expressions 14 and 15.

The asymptotic approximations for utilisation (which are given in the appendix) are highly accurate within the low intensity and high intensity regions. Figure 6 shows that

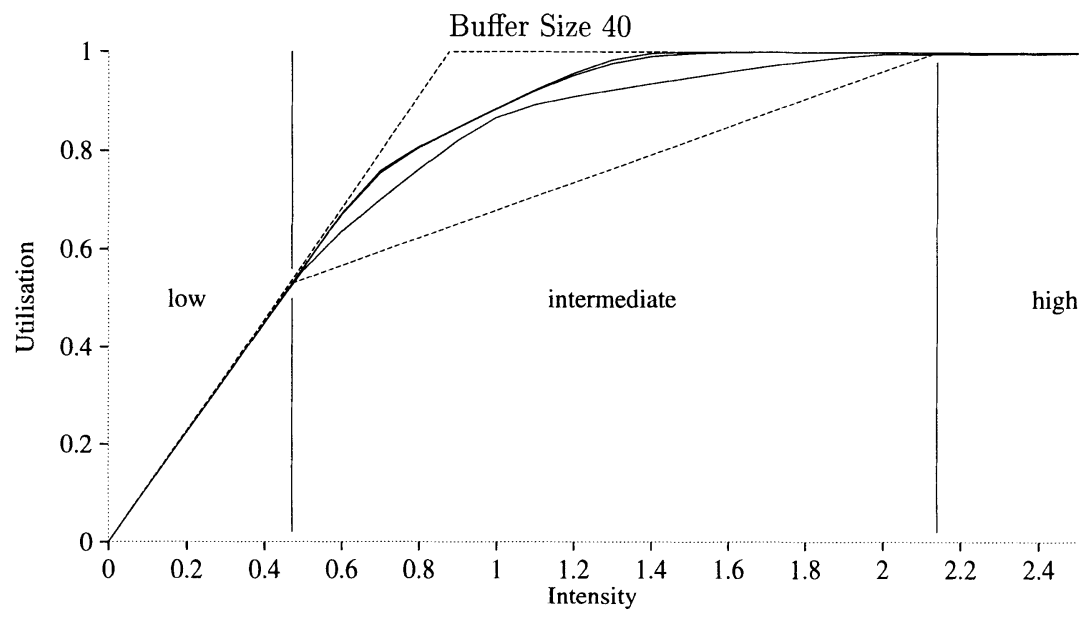

Figure 6. Comparison of fast service and fast arrivals asymptotic approximations for utilisation and heuristic bounds against exact results of figure 4 .

those approximations, when projected into the region of intermediate intensity, are upper bounds to the exact results and that the chord forms a lower bound over almost all of the intermediate intensity region.

\section{Conclusions}

The MMPP/MMPP/1/N systems comprise families in which the behaviour of each of the member systems is practically identical in regions of low intensity and of high intensity. In those regions, simple cost effective accurate approximations may be employed with confidence: suitable approximations are presented in this paper.

For each family, members which exhibit widely different degrees of correlation in arrival or service processes exhibit widely different behaviour in the region of intermediate intensity. The behaviour of such member systems diverges abruptly at the region boundaries. Thus the boundaries are well defined. 
The combination of accurate approximations to system behaviour in the low intensity and high intensity regions together with precise definition of the region boundaries readily leads to simple heuristic bounds on system behaviour in the region of intermediate intensity.

In this paper an asymptotic queueing theoretic method is applied to the performance analysis of a finite capacity, single server queue evolving within a randomly changing environment. The asymptotic method exposes characteristic behaviour which may be obscured in results produced by exact analysis, numerical methods or simulation. The method yields simple closed form expressions for the boundaries of the low intensity and high intensity regions, in which accurate asymptotic approximations may be employed, and implies bounds on system performance in the region of intermediate intensity.

Further research into families of $\mathrm{MMPP} / \mathrm{MMPP} / 1 / \mathrm{N}$ systems is required to discover why there should be such well-defined points from which the behaviour of all members diverges. Further research - based upon both theoretic and heuristic considerations - is also needed to connect the fast arrivals and fast service approximations in order to capture system behaviour in the region of intermediate intensity. Moreover, given the ranges of the parameters from which the analyst must chose, there is need for greater insight into the characterisation and least biased choice parameterisation of the MMPP/MMPP/1/N system and its relationship to simpler and more cost effective models.

Extensions of the work towards analysis of discrete time queueing systems, such as $\mathrm{MMBP} / \mathrm{D} / 1 / \mathrm{N}$ queues $^{6}$, are the subject of current study.

\section{REFERENCES}

1. V.V. Anisimov, J. SzTrik, Asymptotic Analysis of Some Finite-Source Queueing Systems, Acta Cybernetica, Vol. 9, Issue 1, 1989, pp. 27-38

2. V.V. Anisimov, O.K. Zakusilo, V.S. Doncsenko, Elements of Queueing Theory and Asymptotic Analysis of Systems, Visa Skola, Kiev, 1987 (in Russian)

3. János Sztrik, Demetres Kouvatsos, Asymptotic Analysis of a Heterogeneous Multiprocessor System in a Randomly Changing Environment, IEEE Transactions on Software Engineering, Vol. 17, No. 10, October 1991, pp. 1069-1075

4. János SzTrik, Modelling of a Multiprocessor System in a Randomly Changing Environment, Performance Evaluation 17(1993), pp. 1-11

\footnotetext{
${ }^{6} \mathrm{MMBP}=$ Markov Modulated Bernoulli Process.
} 


\section{A. Related Results}

This appendix illustrates some of the results which may be obtained from the asymptotic methods which are described in the main part of the paper. Given below are derivation of queue length distributions and accurate approximations to utilisation which are applicable in the low intensity and high intensity regions.

\section{A.1. Queue Length}

Derivation of theorem 1 utilises an asymptotic expression for the stationary probability $\pi_{\varepsilon}(k)$ that the embedded Markov chain be in state $k, k \in S_{q}, q=1, \ldots, m+1$,

$\pi_{\varepsilon}(k)=\varepsilon^{q} \sum \pi_{0}\left(k_{0}\right) \alpha_{0}\left(k_{0}, k_{1}\right) \ldots \alpha_{q-1}\left(k_{q-1}, k\right)+o\left(\varepsilon^{q}\right)$,

where the summation is over all $k_{0} \in S_{0}, \ldots, k_{q-1} \in S_{q-1}$. This expression is an immediate consequence of condition 1 of theorem 1 . The steady state distribution of the underlying Semi-Markov Process (i.e. the random observer's distribution) may be derived from its relationship with the stationary distribution of the embedded Markov chain and expression 16. However, the derivation of expression 16 suggests a direct approach, which is pursued below.

Expression 16 shows that the asymptotic analysis effectively ignores transitions between states at the same level, except for level 0 (specifically, except for states corresponding to the empty buffer for fast service and except for states corresponding to the full buffer for fast arrivals). If this simplification be applied to the transition rates (equations 1) in the context of fast service, the relationships between the stationary state probabilities $p(n, j, k)$ ( $n$ number in system, arrivals phase $j$, server phase $k$ ) of the underlying SemiMarkov Process are given by

$$
\begin{aligned}
\left(a_{j j}+\lambda_{j}+b_{k k}\right) p(0, j, k) & =\mu_{k} p(1, j, k)+\sum_{l \neq j} a_{l j} p(0, l, k)+\sum_{q \neq k} b_{q k} p(0, j, q) \\
\left(\lambda_{j}+\mu_{k}\right) p(n, j, k) & =\lambda_{j} p(n-1, j, k)+\mu_{k} p(n+1, j, k) \quad n=1, \ldots, N- \\
\mu_{k} p(N, j, k) & =\lambda_{j} p(N-1, j, k)
\end{aligned}
$$

Therefore, a fast service approximation to queue length distribution is given by

$p(n, j, k)=\frac{1}{Z}\left(\frac{\lambda_{j}}{\mu_{k}}\right)^{n} \pi_{A}(j) \pi_{B}(k)$

for all $n=0, \ldots, N, j=1, \ldots, r_{A}, k=1, \ldots, r_{B}$, where $Z$ is the normalising constant

$Z=\sum_{j=1}^{r_{A}} \sum_{k=1}^{r_{B}} \sum_{n=0}^{N}\left(\frac{\lambda_{j}}{\mu_{k}}\right)^{n} \pi_{A}(j) \pi_{B}(k)=\sum_{j=1}^{r_{A}} \sum_{k=1}^{r_{B}} \frac{1-\left(\frac{\lambda_{j}}{\mu_{k}}\right)^{N+1}}{1-\frac{\lambda_{j}}{\mu_{k}}} \pi_{A}(j) \pi_{B}(k)$ by

By similar analysis, a fast arrivals approximation to queue length distribution is given

$p(n, j, k)=\frac{1}{Z}\left(\frac{\mu_{k}}{\lambda_{j}}\right)^{N-n} \pi_{A}(j) \pi_{B}(k)$

for all $n=0, \ldots, N, j=1, \ldots, r_{A}, k=1, \ldots, r_{B}$, where $Z$ is the normalising constant

$Z=\sum_{j=1}^{r_{A}} \sum_{k=1}^{r_{B}} \sum_{n=0}^{N}\left(\frac{\mu_{k}}{\lambda_{j}}\right)^{N-n} \pi_{A}(j) \pi_{B}(k)=\sum_{j=1}^{r_{A}} \sum_{k=1}^{r_{B}} \frac{1-\left(\frac{\mu_{k}}{\lambda_{j}}\right)^{N+1}}{1-\frac{\mu_{k}}{\lambda_{j}}} \pi_{A}(j) \pi_{B}(k)$ 


\section{A.2. Utilisation}

From equation 20 for $n=0$ it follows that a fast service asymptotic approximation to utilisation is given by

$U=1-\frac{1}{Z}$

Also, the intensity $\rho$ is given by

$\rho=\frac{\sum_{j=1}^{r_{A}} \pi_{A}(j) \lambda_{j}}{\sum_{k=1}^{r_{B}} \pi_{B}(k) \mu_{k}}$

Hence, for low intensity, from equations 21 and 25 an asymptotic expression for $Z$ is given by

$Z=1+\left(\sum_{k=1}^{r_{B}} \pi_{B}(k) \mu_{k}\right)\left(\sum_{k=1}^{r_{B}} \frac{\pi_{B}(k)}{\mu_{k}}\right) \rho+o(\rho)$

and so, from equations 24 and 26, a fast service asymptotic approximation to utilisation is given by

$$
\begin{aligned}
U & =1-\frac{1}{1+\left(\sum_{k=1}^{r_{B}} \pi_{B}(k) \mu_{k}\right)\left(\sum_{k=1}^{r_{B}} \frac{\pi_{B}(k)}{\mu_{k}}\right) \rho}+o(\rho) \\
& =\left(\sum_{k=1}^{r_{B}} \pi_{B}(k) \mu_{k}\right)\left(\sum_{k=1}^{r_{B}} \frac{\pi_{B}(k)}{\mu_{k}}\right) \rho+o(\rho)
\end{aligned}
$$

It is notable that this fast service asymptotic approximation to utilisation is independent of the parameters of the arrival process.

The accuracy of the approximation is shown below by comparison (to 5 significant digits) with the exact results which are illustrated in figures 4 and 5 of section 6 .

By similar analysis, a fast arrivals approximation to utilisation is given by

$$
U=1+o\left(\frac{1}{\rho^{N-1}}\right)
$$


Buffer Size 10

\begin{tabular}{|c|c|ccc|ccc|}
\hline Intensity & Approximation & \multicolumn{3}{|c|}{ figure 4 $(L \approx .36)$} & \multicolumn{3}{c|}{ figure 5 $(L \approx .25)$} \\
$\rho$ & expr.(26) & line 1 & line 2 & line 3 & line 1 & line 2 & line 3 \\
\hline 0.1 & .1125 & .11247 & .11247 & .11247 & .11250 & .11250 & .11250 \\
0.2 & .2250 & .22490 & .22490 & .22491 & .22464 & .22465 & .22490 \\
0.3 & .3375 & .33692 & .33717 & .33727 & .32619 & .32642 & .33441 \\
0.4 & .4500 & .44503 & .44795 & .44892 & .39235 & .39330 & .43016 \\
0.5 & .5625 & .53985 & .55224 & .55641 & .43409 & .43565 & .50486 \\
\hline
\end{tabular}

Buffer Size 40

\begin{tabular}{|c|c|ccc|ccc|}
\hline Intensity & Approximation & \multicolumn{3}{|c|}{ figure $4(L \approx .47)$} & \multicolumn{3}{c|}{ figure $5(L \approx .31)$} \\
$\rho$ & expr. $(26)$ & line 1 & line 2 & line 3 & line 1 & line 2 & line 3 \\
\hline 0.1 & .1125 & .11247 & .11247 & .11247 & .11250 & .11250 & .11250 \\
0.2 & .2250 & .22490 & .22491 & .22491 & .22500 & .22500 & .22500 \\
0.3 & .3375 & .33727 & .33729 & .33731 & .33717 & .33724 & .33750 \\
0.4 & .4500 & .44937 & .44956 & .44962 & .40063 & .40717 & .44977 \\
0.5 & .5625 & .55680 & .56147 & .56167 & .43838 & .44670 & .55687 \\
\hline
\end{tabular}

Figure 7. Comparison of fast service asymptotic approximations for utilisation against exact results for buffer sizes 10 and 40. Results in italics are for values of intensity outside the range of validity for the approximation, i.e. for $\rho>L$. 